\title{
Are water-xylitol mixtures heterogeneous? An investigation employing composition and temperature dependent dielectric relaxation and time-resolved fluorescence measurements
}

\author{
EJAJ TARIF ${ }^{\mathrm{a}}$, KALLOL MUKHERJEE ${ }^{\mathrm{a}}$, ANJAN BARMAN ${ }^{\mathrm{b}}$ and RANJIT BISWAS ${ }^{\mathrm{a}, *}$ \\ ${ }^{a}$ Chemical, Biological and Macromolecular Sciences (CBMS), S N Bose National Centre for Basic Sciences, \\ JD Block, Sector III, Salt Lake, Kolkata 700 106, West Bengal, India \\ ${ }^{\mathrm{b}}$ Condensed Matter Physics and Materials Science, S N Bose National Centre for Basic Sciences, JD Block, \\ Sector III, Salt Lake, Kolkata 700 106, West Bengal, India \\ E-mail: ranjit@bose.res.in
}

MS received 22 January 2019; revised 2 March 2019; accepted 6 March 2019; published online 10 May 2019

\begin{abstract}
Aqueous xylitol solutions at six different concentrations were studied employing dielectric relaxation (DR) and time-resolved fluorescence (TRF) measurements in the temperature range 295-323 K. The focus was to explore the solution heterogeneity aspect via monitoring the viscosity coupling of the average relaxation rates at various temperatures. TRF measurements were done using both hydrophobic and hydrophilic probes to explore the preferences, if any, for solute locations in these binary mixtures. Energy-selective population excitations and the corresponding fluorescence emissions did not suggest any significant spatial heterogeneity in solution structure within the lifetimes of these probes. DR measurements and TRF experiments indicated mild deviations from the hydrodynamic viscosity dependence of the measured relaxation rates. All these suggest mild spatiotemporal heterogeneity for these water-xylitol mixtures in the temperature range considered. In addition, DR timescales appear to originate from reorientational and $\mathrm{H}$-bond relaxation dynamics, excluding the possibility of full molecular rotations.
\end{abstract}

Keywords. Xylitol; water; heterogeneity; dielectric relaxation; fluorescence.

\section{Introduction}

Biologically important molecules, especially the building blocks of cell walls, nucleic acids, exoskeletons and also the regulators of the human body's functions, constitute an important area of research. A thorough understanding of microscopic interaction and dynamics in the aqueous phase is necessary for a smarter application of biologically relevant molecules, such as amino acids, saccharides, polyols, etc. Xylitol is a polyhydroxy alcohol which contains five hydroxyl groups attached to five separate carbon atoms and is represented by the chemical formula, $\mathrm{CH}_{2} \mathrm{OH}(\mathrm{CHOH})_{3} \mathrm{CH}_{2} \mathrm{OH}$. Xylitol is considered as natural sugar because it is found in many vegetables, fruits, and also produced in the human metabolism process. ${ }^{1}$ Pure xylitol is sweet as sugar and is believed to reduce dental plaque, caries, and assists in remineralization of teeth. ${ }^{2-4}$ Lower glycemic-index value (GI 7) of xylitol makes it a potential alternative to commonly used sugar (glucose GI 100) for diabetic patients. ${ }^{5,6}$ Xylitol, like many other polyols, is widely used as a food additive due to non-carcinogenicity, low energy content, and other relevant features. ${ }^{5,7,8}$

Structure and dynamics of pure water undergo considerable changes in the presence of external solutes and co-solvents (such as sugar, alcohol, etc.). ${ }^{9-13}$ Identification of the origins for such changes in solute-solvent mixtures is an important aspect. Water-sugar and/or water-polyol mixtures are critically relevant to processes that sustain life and assume importance in food sciences and cryopreservation technology. In water-sugar or water-polyol mixtures, the presence of hydrophilic and hydrophobic interactions add to the complexity in the structural and dynamical properties of the solution via resolving the interparticle interactions in a new way and modifying the motional features of the mixture

\footnotetext{
*For correspondence 
components. Aqueous solutions of sugar or polyol are known to stabilize proteins and other biological substances. ${ }^{14-16}$ The stabilization of proteins may occur via either changing their internal structural characteristics or altering the external medium properties that are in contact with them. It is believed that stabilization (by external stimuli) of protein occurs through processes such as preferential solvation, alteration of water structure, etc. ${ }^{17-19}$ Sometimes it may be assisted by the solution-phase spatiotemporal heterogeneity. ${ }^{20}$ Thus, a thorough knowledge of the structure and dynamics of aqueous solutions containing sugar or other polyols is critical for understanding the activity of biologically relevant molecules in aqueous environments, and their preservation at cryogenic temperatures.

Relaxation in glass-forming liquids (such as sugar, polyols) has been studied by using various techniques (such as dielectric relaxation, light scattering, etc.). ${ }^{21-24}$ Notably, these studies explore the impact of water on glass transition temperature as well as relaxation behavior. Interestingly, in many glass-forming liquids an ultraslow dynamics was detected in dielectric relaxation (DR), ${ }^{25-28}$ and dynamic light scattering (DLS) ${ }^{29,30}$ measurements. This ultraslow process is different from the viscosity related structural $(\alpha)$ relaxation and was explained by long-range density fluctuations or hydrogen bonded cluster diffusion. A low-frequency Debye peak in the imaginary part of the permittivity is a characteristic representation of an ultraslow process. This Debye peak has been observed in alcohols and their mixtures which represents slower dynamics than the viscosity-related $(\alpha)$ relaxation. ${ }^{25,26}$ It is to be noted that, low frequency Debye peak has been observed in xylitol and supports the presence of ultraslow process other than viscosity related structural $(\alpha)$ relaxation. ${ }^{31}$ Although ultraslow relaxation processes in water-xylitol mixtures were detected in dynamic light scattering (DLS) measurements, small-angle neutron scattering (SANS) experiments did not find any significant excess scattering or any structural inhomogeneity in the medium. ${ }^{32}$ These SANS results, therefore, raise a debate regarding the interpretation of ultra-slow relaxation detected in DLS measurements in terms of cluster formation. Most of these studies either focused on slow dynamics of xylitol $^{21,27,31}$ or relaxations of water-xylitol mixtures ${ }^{22,33}$ at lower temperatures. Though, there exist a few sporadic studies $^{24,32}$ at higher temperatures ( $>298 \mathrm{~K}$ ), a thorough and uniform study of dynamics and interaction in water-xylitol mixtures addressing the solution heterogeneity aspect at temperatures higher than room temperature is still lacking.

We address the solution heterogeneity aspect in this paper via steady-state and time-resolved fluorescence measurements, and DR experiments in the temperature range, $295-323 \mathrm{~K}$. Temperatures beyond $323 \mathrm{~K}$ have not been considered because (i) we wanted to explore the solution characteristics at a temperature range not too away from the physiological temperature $(\sim 310 \mathrm{~K})$ given the fact that xylitol is produced during metabolism, (ii) the DR dynamics becomes faster at higher temperature, particularly those at lower xylitol concentrations, which eventually become undetectable in our frequency window, and (iii) the heterogeneity signature becomes weaker upon increasing temperature. We presume that extensive interaction of water molecules with xylitol may lead to orientational relaxation slower than bulk neat water, and this may be detected in the present DRS measurements. In addition, exploration of the viscosity coupling to solute and solventcentred dynamics would lead to qualitative information regarding micro-heterogeneous nature of these solutions. In this work, measurements have been carried out for water-xylitol mixtures in various concentrations (2.31 mol\% to $9.62 \mathrm{~mol} \%$ ) of xylitol. DRS technique has already been used to understand the dynamics of pure solvents, ${ }^{34-37}$ water-alcohol mixtures, ${ }^{13,38}$ deep eutectic solvents (DESs) ${ }^{39,40}$ and other media. TRF measurements of non-reactive solution dynamics and DR is intimately related, and a combination of them has been employed to explore dynamics and interaction in many different systems. ${ }^{11,41-44}$ For fluorescence measurements, we have used non-reactive hydrophilic coumarin 343 (C343) and hydrophobic coumarin 153 $(C 153)$ as external probes to profile the medium frictional response on a dissolved solute. We refrained from measuring the solvation dynamics (via dynamic Stokes shift measurements) of these probes in these mixtures as water response is too fast to be detected by the present setup (Section 2). Chemical structures of xylitol, coumarin 343 and coumarin 153 are shown in Scheme 1.

\section{Experimental}

\subsection{Sample preparation}

Laser-grade coumarin 153 (C153) and coumarin 343 (C343) were from Sigma-Aldrich and used as received. Xylitol was from Sisco Research Laboratories (SRL, India) and used as received. Solutions of six different concentrations of xylitol were prepared by dissolving the required amount of xylitol (by weight) in millipore water at room temperature. Stock solutions of $C 153$ and $C 343$ were prepared in carrier solvents, such as heptane and acetone, respectively. A few $\mu \mathrm{L}$ of these stock solutions were taken into quartz cuvettes (optical path length $1 \mathrm{~cm}$ ), and the career solvent evaporated off. Approximately $3 \mathrm{~mL}$ of sample solution (water + xylitol) 


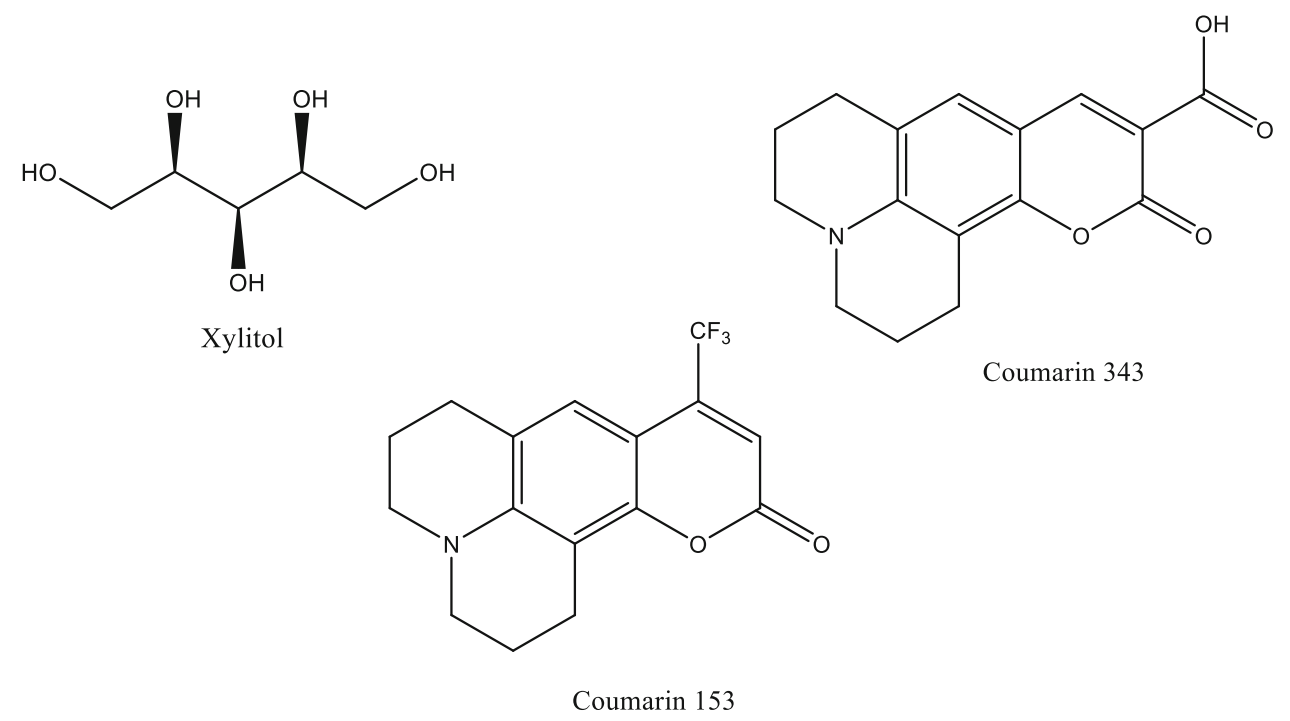

Scheme 1. Chemical structures of xylitol, coumarin 343, and coumarin 153.

was then poured into the cuvette and, complete dissolution of $C 153 / C 343$ grains in sample solution ensured. The concentration of $C 153$ (or $C 343$ ) in each of these sample solutions were maintained at $\sim 10^{-5} \mathrm{M}$.

\subsection{Viscosity and refractive index measurements}

Temperature-dependent viscosity coefficient and the refractive index of water-xylitol mixtures were measured by using AMVn automated micro-viscometer from Anton Paar (falling ball method) and automated temperature controlled refractometer (RUDOLPH, J357), respectively. ${ }^{43,45,46}$

\subsection{Steady-state and time-resolved fluorescence measurements}

Steady-state absorption and emission spectra were collected using a UV-visible spectrophotometer (UV-2600, Shimadzu) and a fluorimeter (Fluorolog, JobinYvon, Horiba), respectively, and data analysis was carried out following the protocol described elsewhere. ${ }^{44,47-49}$

Time-resolved fluorescence measurements were performed using a time-correlated single photon counting (TCSPC) (LifeSpecps, Edinburgh Instruments, U. K.) setup fitted with a diode laser of $409 \mathrm{~nm}$ wavelength (details provided elsewhere). ${ }^{50-52}$ The instrument response function (IRF) measured using scattering solution was found to be $\sim 85$ ps. Time-resolved fluorescence anisotropy $(r(t))$ measurements were performed at the peak wavelength of the steady-state emission spectrum as usual and $r(t)$ were determined from the well-known formula ${ }^{10,53-55}$

$r(t)=\frac{I_{\text {para }}(t)-G I_{\text {perp }}(t)}{I_{\text {para }}(t)+2 G I_{\text {perp }}(t)}$.

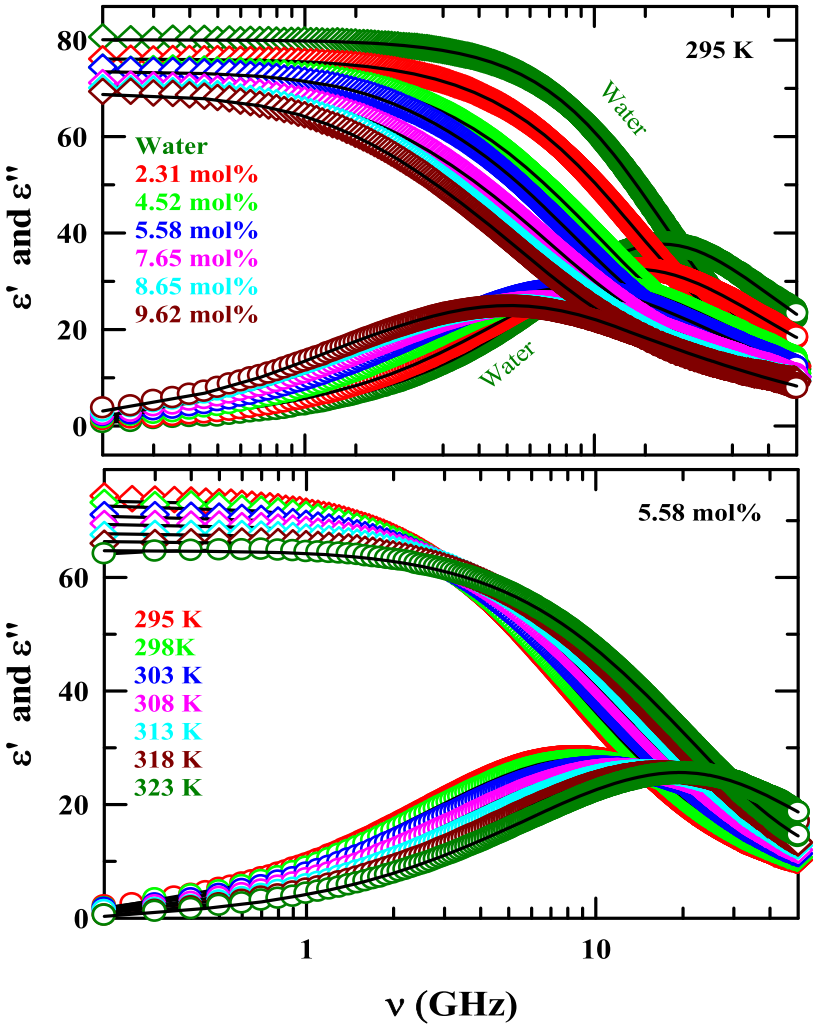

Figure 1. Upper panel: DR spectra of water-xylitol mixtures at $295 \mathrm{~K}$ within the frequency regime, $0.2 \leq v / \mathrm{GHz} \leq 50$ at various xylitol concentrations. Lower panel: Temperature dependence of the real $\left(\varepsilon^{\prime}\right)$ and imaginary $\left(\varepsilon^{\prime \prime}\right)$ parts of the measured complex DR spectra of water-xylitol $(5.58 \mathrm{~mol} \%)$. Solid lines through data represent simultaneous fits using multi-Debye relaxation model. Spectra at different xylitol concentrations and different temperatures are color-coded. Green color represents DR response of neat water. 
Table 1. Parameters obtained from the 3-D/2-D fits of the complex dielectric response functions of water-xylitol mixtures for all the concentrations at $295 \mathrm{~K}$.

\begin{tabular}{lcccccccccccc}
\hline Mole \% Xylitol & $\mathrm{T}(\mathrm{K})$ & $\varepsilon_{0}$ & $\Delta \varepsilon_{1}^{a} \%$ & $\tau_{1}^{b}(\mathrm{ps})$ & $\Delta \varepsilon_{2} \%$ & $\tau_{2}(\mathrm{ps})$ & $\Delta \varepsilon_{3} \%$ & $\tau_{3}(\mathrm{ps})$ & $\varepsilon_{\infty}$ & $n_{D}^{c}$ & $\varepsilon_{\infty}-n_{D}^{2}$ & $\left\langle\tau_{a v}\right\rangle(\mathrm{ps})$ \\
\hline Water & 295 & 80.1 & - & - & 100 & 9.3 & - & - & 4.7 & 1.333 & 2.92 & 9.3 \\
2.31 & 295 & 76.4 & 7.6 & 48 & 67.8 & 13 & 24.6 & 6.6 & 6.1 & 1.358 & 4.26 & 14 \\
& 298 & 76.1 & 5.7 & 46 & 51.5 & 15 & 42.8 & 7.9 & 6.6 & 1.358 & 4.76 & 14 \\
& 303 & 73.7 & 2.7 & 51 & 25.3 & 17 & 72.6 & 7.6 & 5.9 & 1.359 & 4.05 & 11 \\
& 308 & 72.5 & 2.6 & 47 & 21.6 & 16 & 75.5 & 7.2 & 5.7 & 1.359 & 3.85 & 10 \\
& 313 & 72.3 & - & - & 15.5 & 16 & 84.5 & 6.7 & 4.8 & 1.359 & 2.95 & 8 \\
4.52 & 318 & 70.2 & - & - & 14.1 & 19 & 85.9 & 6.5 & 4.9 & 1.360 & 3.05 & 8 \\
5.58 & 323 & 68.9 & - & - & 13.9 & 16 & 86.1 & 5.8 & 3.9 & 1.360 & 2.05 & 7 \\
& 295 & 73.9 & 15.7 & 49 & 66.4 & 16 & 17.9 & 6.2 & 6.8 & 1.378 & 4.90 & 19 \\
& 295 & 73.4 & 20.7 & 57 & 63.5 & 18 & 15.8 & 5.7 & 6.7 & 1.386 & 4.78 & 24 \\
& 298 & 72.7 & 18.7 & 56 & 62.0 & 18 & 19.3 & 6.4 & 7.1 & 1.386 & 5.18 & 23 \\
& 303 & 70.8 & 15.4 & 55 & 52.9 & 19 & 31.7 & 6.9 & 7.0 & 1.386 & 5.08 & 21 \\
& 308 & 69.4 & 11.9 & 54 & 47.8 & 18 & 40.3 & 7.1 & 6.9 & 1.386 & 4.97 & 18 \\
7.65 & 313 & 67.8 & 9.0 & 52 & 41.2 & 18 & 49.8 & 6.9 & 6.7 & 1.385 & 4.78 & 15 \\
8.65 & 318 & 66.4 & 5.7 & 58 & 35.9 & 18 & 58.4 & 6.8 & 6.5 & 1.386 & 4.57 & 13 \\
9.62 & 323 & 64.7 & 4.8 & 55 & 30.7 & 17 & 64.5 & 6.3 & 6.1 & 1.386 & 4.18 & 12 \\
& 295 & 70.7 & 25.1 & 68 & 60.6 & 21 & 14.3 & 6.0 & 6.9 & 1.399 & 4.94 & 31 \\
& 295 & 70.1 & 29.8 & 77 & 55.7 & 24 & 14.5 & 6.7 & 7.0 & 1.405 & 5.02 & 37 \\
& 295 & 68.9 & 31.7 & 80 & 54.8 & 26 & 13.6 & 5.6 & 7.1 & 1.410 & 5.11 & 40 \\
& 298 & 67.9 & 28.6 & 78 & 53.2 & 28 & 18.2 & 6.6 & 7.2 & 1.410 & 5.21 & 38 \\
& 303 & 66.2 & 25.0 & 76 & 52.3 & 25 & 22.7 & 6.6 & 7.2 & 1.410 & 5.21 & 34 \\
& 308 & 65.5 & 23.6 & 69 & 51.3 & 23 & 25.1 & 6.6 & 7.3 & 1.410 & 5.31 & 30 \\
& 313 & 63.7 & 18.6 & 64 & 49.9 & 22 & 31.5 & 6.6 & 7.3 & 1.410 & 5.31 & 25 \\
& 318 & 62.4 & 16.5 & 58 & 47.6 & 20 & 35.9 & 6.4 & 7.1 & 1.410 & 5.11 & 21 \\
& 323 & 61.8 & 16.1 & 50 & 47.4 & 18 & 36.5 & 6.0 & 7.0 & 1.410 & 5.01 & 19 \\
\hline
\end{tabular}

(a) Indicates dispersion amplitude $\left(\Delta \varepsilon_{i}, i=1-3\right)$ of a given dispersion step in percentage.

(b) $\tau_{i}(i=1-3)$ are better within $\pm 5 \%$ of the reported values (based on 2-3 independent measurements).

(c) Measured refractive index at $295 \mathrm{~K}$.

Where $I_{\text {para }}(t)$ and $I_{\text {perp }}(t)$ are parallel and perpendicular decays, respectively. The factor accounts for the differential sensitivity to the two polarization and was obtained ( $G=1.45 \pm 0.1$ ) by the tail matching of the intensity decays $I_{\text {para }}(t)$ and $I_{\text {perp }}(t)$. Average rotational time was obtained via time integrating the normalized $r(t)$ decay: $\left\langle\tau_{r}\right\rangle=$ $\int_{0}^{\infty} d t\left[r(t) / r_{0}\right]$. The value of initial anisotropy, $r_{0}$, was used as $0.376^{53}$ for $C 153$ and $0.35^{56}$ for $C 343$. In the present anisotropy measurements, $r(t)$ decays were found to be single-exponential functions of time for both $\mathrm{C} 153$ and $\mathrm{C} 343$ in these aqueous solutions, and thus, $\left\langle\tau_{\mathrm{r}}\right\rangle=\int_{0}^{\infty} \mathrm{dt}\left[\mathrm{r}(\mathrm{t}) / \mathrm{r}_{0}\right]=$ $\int_{0}^{\infty} \mathrm{dt}[\mathrm{a} \exp (-\mathrm{t} / \tau)]=\mathrm{a} \tau$.

\subsection{Dielectric relaxation spectroscopy}

The frequency dependent complex relative permittivity $\varepsilon^{*}(v)$ is expressed as ${ }^{57}$

$\varepsilon^{*}(v)=\varepsilon^{\prime}(v)-\left[i \varepsilon^{\prime \prime}(v)+\frac{i \kappa}{2 \pi v \varepsilon_{P}}\right]$.
Here, $\kappa$ is the dc conductivity of the medium, $\varepsilon_{p}$ the permittivity of free space. $\varepsilon^{\prime}$ and $\varepsilon^{\prime \prime}$ represents the real and imaginary components of the complex permittivity, respectively. Dielectric spectra were collected using a PNA-L Network Analyzer (N5230C) combined with a probe kit (85070E) operating in the frequency range $0.2 \leq \mathrm{GHz} \leq 50$. Around $8-10 \mathrm{~mL}$ solution of each mixture was used for all the measurements. Details regarding DR measurements can be found elsewhere. ${ }^{40}$ Experimentally obtained frequency dependent complex relative permittivity $\varepsilon^{*}(\nu)$ was then fitted with a sum of $n$ Havriliak-Negami (HN) equation. ${ }^{57}$

$\varepsilon^{*}(\nu)=\varepsilon_{\infty}+\sum_{j=1}^{n} \frac{\Delta \varepsilon_{j}}{\left[1+\left(i 2 \pi \nu \tau_{j}\right)^{1-\alpha_{j}}\right]^{\beta_{j}}}$,

where $0 \leq \alpha_{j}<1$ and $0<\beta_{j} \leq 1 . \Delta \varepsilon_{j}$ represents the dispersion magnitude at the $j-$ th relaxation step with the time constant, $\tau_{j}$. Debye (D) relaxation corresponds to $\alpha_{j}=0$, $\beta_{j}=1$ whereas $\alpha_{j}=0$ describes the Cole-Davidson (CD) and $\beta_{j}=1$ the Cole-Cole (CC) models respectively. Simultaneous fitting of $\varepsilon^{\prime}$ and $\varepsilon^{\prime \prime}$ by using a non-linear least squares method produced the relaxation parameters that described the measured spectra adequately. For accurate description, sufficient numbers of data points were collected during 


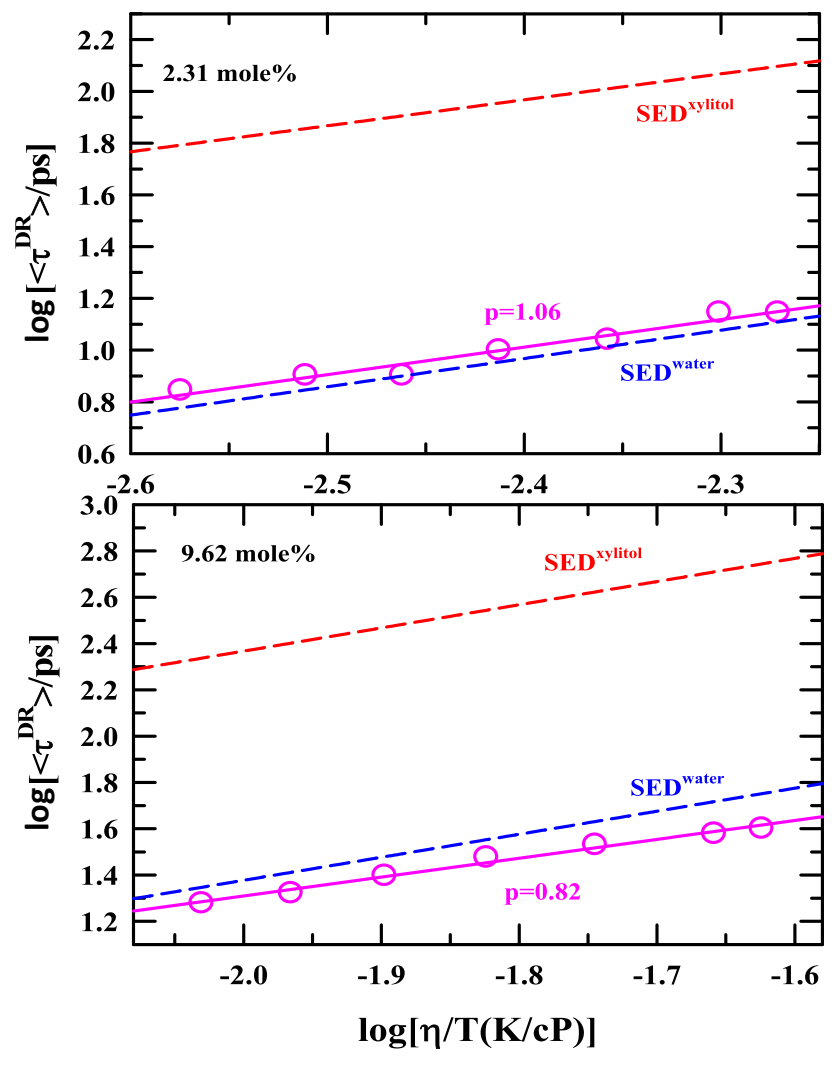

Figure 2. $(\eta / T)$ dependence of the average DR relaxation times $\left(\left\langle\tau_{\mathrm{DR}}\right\rangle\right)$ for aqueous xylitol solutions at the lowest (upper panel) and the highest (lower panel) concentrations. SED predictions with stick boundary condition for water and xylitol molecules using the experimental temperature dependent solution viscosity coefficients ( $\eta$, see Table 2 ) are also shown in these panels for comparison.

measurements within the available frequency window. Both the 'goodness-of-fit' parameter $\left(\chi^{2}\right)$ and residuals were checked for ensuring the quality of fits. The following expres$\operatorname{sion}^{58}$ defines the $\left(\chi^{2}\right)$

$\chi^{2}=\frac{1}{2 m-\ell} \sum_{i=1}^{m}\left[\left(\frac{\delta \varepsilon_{i}^{\prime}}{\sigma\left(\varepsilon_{i}^{\prime}\right)}\right)^{2}+\left(\frac{\delta \varepsilon_{i}^{\prime \prime}}{\sigma\left(\varepsilon_{i}^{\prime \prime}\right)}\right)^{2}\right]$

With $m$ representing the number of data triples $\left(\nu, \varepsilon^{\prime}, \varepsilon^{\prime \prime}\right)$, $\ell$ the number of adjustable parameters, $\delta \varepsilon_{i}$ and $\sigma\left(\varepsilon_{i}\right)$ the residuals and standard deviations of the individual data points, respectively.

Among all measurements presented here, some of the DR spectra fit to 2-D (2-Debye) and rest to 3-D (3-Debye) relaxations. Fits were employed to obtain the best simultaneous descriptions of both the measured $\varepsilon^{\prime}(v)$ and $\varepsilon^{\prime \prime}(v)$. Different combinations of Debye, Cole-Cole and Cole-Davidson processes were attempted but did not obtain any better description than the fits chosen here (shown in Figure S1, Supplementary Information).

\section{Results and Discussion}

\subsection{Dielectric relaxation measurements: concentration and temperature dependence}

Figure 1 presents the concentration and temperature dependent real $\left(\varepsilon^{\prime}\right)$ and imaginary $\left(\varepsilon^{\prime \prime}\right)$ components of the measured complex dielectric relaxation (DR) spectra of water-xylitol mixtures along with simultaneous multi-Debye fits. Concentration-dependent measurements were done at $295 \mathrm{~K}$ and at six different xylitol concentrations ( $\mathrm{mol} \%)$. The highest concentration chosen here is limited by the aqueous solubility of xylitol at 295 K. For comparison, we have also shown our experimental DR spectra of pure water at $295 \mathrm{~K}$ in the same (upper) panel. Fit parameters are summarized in Table 1. Two aspects could be immediately realized from these concentration-dependent spectra. The first observation is the gradual decrease of the estimated static dielectric constant $\left(\varepsilon_{0}\right)$ upon increase of xylitol concentration in the aqueous solution. Second, the peak position in the imaginary component $\left(\varepsilon^{\prime \prime}\right)$ shifts to a lower frequency with xylitol concentration, producing longer relaxation times at higher concentrations. The concentrationdependent slowest DR timescale $\left(\tau_{1}\right)$ falls in $\sim 50-80 \mathrm{ps}$ range, the fastest $\left(\tau_{3}\right)$ being $<10 \mathrm{ps}$. Another timescale $\left(\tau_{2}\right)$ also appears at this temperature which is somewhat slower than the fastest but covers the range $\sim 13-26 \mathrm{ps}$.

The decrease of $\varepsilon_{0}$ with xylitol concentration is expected because $\varepsilon_{0}$ of xylitol is $\sim 40 .{ }^{59}$ Note DR measurements with appropriate frequency coverage for neat water have revealed two relaxation timescales $(\sim 9 \mathrm{ps}$ and $\sim 1 \mathrm{ps}$ ) in pure water at $\sim 293 \mathrm{~K} .{ }^{60}$ We also have observed the $\sim 9$ ps timescale in our DR measurements for pure water at $295 \mathrm{~K}$, although we have missed the fast $1 \mathrm{ps}$ timescale, probably due to our limited frequency coverage at the high-frequency wing (up to $50 \mathrm{GHz}$ only). We may, therefore, associate the $<10$ ps DR timescale observed for xylitol solutions with the DR of bulk-like water molecules. The other two DR timescales ( $\tau_{1}$ and $\left.\tau_{2}\right)$ are much slower than the DR timescale of bulk pure water and thus may have a connection to xylitol orientation dynamics. The slowest timescale $\left(\tau_{1} \sim 48-80 \mathrm{ps}\right)$ and its magnitude (8-32\%) increases with increasing xylitol concentration and therefore supports the connection of xylitol molecules to the slow (compared to neat water) DR dynamics in these aqueous mixtures. Notably, the second slower component $\left(\tau_{2}\right)$ dominates the total relaxation $(\sim 68-55 \%)$ and also becomes longer with xylitol concentration.

Now, what could be the likely origins for these two slower timescales, $\tau_{1}$ and $\tau_{2}$ ? In water-xylitol mixtures, 
Table 2. Viscosity, refractive indices and average rotational time $\left\langle\tau_{r}\right\rangle$ of water-xylitol mixtures at different mole $\%$ of xylitol and temperatures.

\begin{tabular}{|c|c|c|c|c|c|}
\hline Xylitol mol\% & $\mathrm{T}(\mathrm{K})$ & $\eta(\mathrm{cP})$ & $\left\langle\tau_{r}\right\rangle(\mathrm{ps})[\mathrm{C} 153]$ & $\left\langle\tau_{r}\right\rangle(\mathrm{ps})[\mathrm{C} 343]$ & Density $\left(\mathrm{g} / \mathrm{cm}^{3}\right)$ \\
\hline \multirow[t]{6}{*}{2.31} & 298 & 1.49 & 143 & 143 & 1.0537 \\
\hline & 303 & 1.33 & 132 & 127 & 1.0519 \\
\hline & 308 & 1.19 & 115 & 119 & 1.0498 \\
\hline & 313 & 1.08 & 95 & 100 & 1.0475 \\
\hline & 318 & 0.98 & 87 & 91 & 1.0441 \\
\hline & 323 & 0.86 & 83 & 84 & 1.0407 \\
\hline \multirow[t]{6}{*}{4.52} & 298 & 2.29 & 201 & 195 & 1.0963 \\
\hline & 303 & 2.04 & 183 & 174 & 1.0941 \\
\hline & 308 & 1.87 & 160 & 166 & 1.0918 \\
\hline & 313 & 1.63 & 130 & 129 & 1.0886 \\
\hline & 318 & 1.44 & 119 & 115 & 1.0853 \\
\hline & 323 & 1.30 & 108 & 109 & 1.0813 \\
\hline \multirow[t]{6}{*}{5.58} & 298 & 2.92 & 222 & 263 & 1.1153 \\
\hline & 303 & 2.52 & 189 & 215 & 1.1130 \\
\hline & 308 & 2.26 & 157 & 180 & 1.1105 \\
\hline & 313 & 1.96 & 148 & 164 & 1.1078 \\
\hline & 318 & 1.75 & 138 & 150 & 1.1049 \\
\hline & 323 & 1.60 & 125 & 120 & 1.1019 \\
\hline \multirow[t]{6}{*}{7.65} & 298 & 4.35 & 335 & 350 & 1.1479 \\
\hline & 303 & 3.71 & 280 & 304 & 1.1453 \\
\hline & 308 & 3.19 & 222 & 251 & 1.1427 \\
\hline & 313 & 2.78 & 207 & 190 & 1.1391 \\
\hline & 318 & 2.44 & 170 & 168 & 1.1370 \\
\hline & 323 & 2.16 & 158 & 150 & 1.1339 \\
\hline \multirow[t]{6}{*}{8.65} & 298 & 5.35 & 354 & 399 & 1.1616 \\
\hline & 303 & 4.50 & 292 & 357 & 1.1590 \\
\hline & 308 & 3.87 & 268 & 298 & 1.1561 \\
\hline & 313 & 3.35 & 243 & 252 & 1.1529 \\
\hline & 318 & 2.90 & 180 & 205 & 1.1484 \\
\hline & 323 & 2.55 & 166 & 173 & 1.1449 \\
\hline \multirow[t]{6}{*}{9.62} & 298 & 6.54 & 458 & 445 & 1.1741 \\
\hline & 303 & 5.45 & 360 & 357 & 1.1718 \\
\hline & 308 & 4.62 & 280 & 310 & 1.1686 \\
\hline & 313 & 3.96 & 245 & 298 & 1.1658 \\
\hline & 318 & 3.44 & 203 & 256 & 1.1626 \\
\hline & 323 & 3.01 & 180 & 196 & 1.1580 \\
\hline
\end{tabular}

it is quite natural to expect that the relaxation dynamics would be regulated by both $\mathrm{H}$-bonding fluctuation dynamics and orientation relaxations. ${ }^{61}$ Interestingly, the magnitudes of $\tau_{2}(\sim 13-26 \mathrm{ps})$ corroborate well with the concentration-dependent peak times corresponding to the peak frequencies in $\varepsilon^{\prime \prime}$ displayed in the upper panel ( $\tau_{\text {peak }}=1 / 2 \pi v_{\text {peak }}$, with $v_{\text {peak }}^{2.31 \text { mol } \%} \sim 15 \mathrm{GHz}$ producing $\sim 12 \mathrm{ps}$, and $v_{\text {peak }}^{9.62 \mathrm{~mol} \%} \sim 5 \mathrm{GHz}$ producing $\sim 32 \mathrm{ps}$ ). Stokes-Einstein-Debye (SED) ${ }^{53,62-64}$ relation with the stick boundary condition, $\tau_{r}=3 \eta V / k_{B} T$, predicts values of molecular rotation times for xylitol and water at $295 \mathrm{~K}$ in these solutions either to be too large or inconsistent to be favorably compared to the observed $\tau_{1}$ and $\tau_{2}$ or to their amplitude-weighted average, $\left\langle\tau_{D R}\right\rangle=\sum_{i=1}^{2} a_{\mathrm{i}} \tau_{\mathrm{i}}$. Table S1 (Supplementary Information) provides this comparison after connecting $\tau_{\mathrm{r}}$ with $\tau_{\mathrm{DR}}$ as follows, $\tau_{\mathrm{r}}=\frac{\ell(\ell+1)}{2} \times \tau_{\mathrm{DR}}$, and for DR, $\ell=1$. Molecules were treated as spheres in the SED predictions with molecular volumes $(\mathrm{V})$ for water ${ }^{65,66}$ as $10.9 \AA^{3}$ and $107.3 \AA^{3}$ for xylitol. ${ }^{67}$ It is therefore quite clear that molecular rotation times cannot cogently explain these two relaxation times. In light of the recent findings for acetamide containing deep eutectics,${ }^{61}$ these components may derive contributions from $\mathrm{H}$-bond fluctuation dynamics and collective single particle reorientational relaxations. Simulation studies are therefore required to confirm this conjecture.

The temperature dependent ( $295 \mathrm{~K}$ to $323 \mathrm{~K}$ ) DR spectra shown in the lower panel (Figure 1) are a representative of the DR measurements that we have carried out for three $(2.31,5.58$ and $9.62 \mathrm{~mol} \%)$ of the six different xylitol concentrations considered here. Here also 


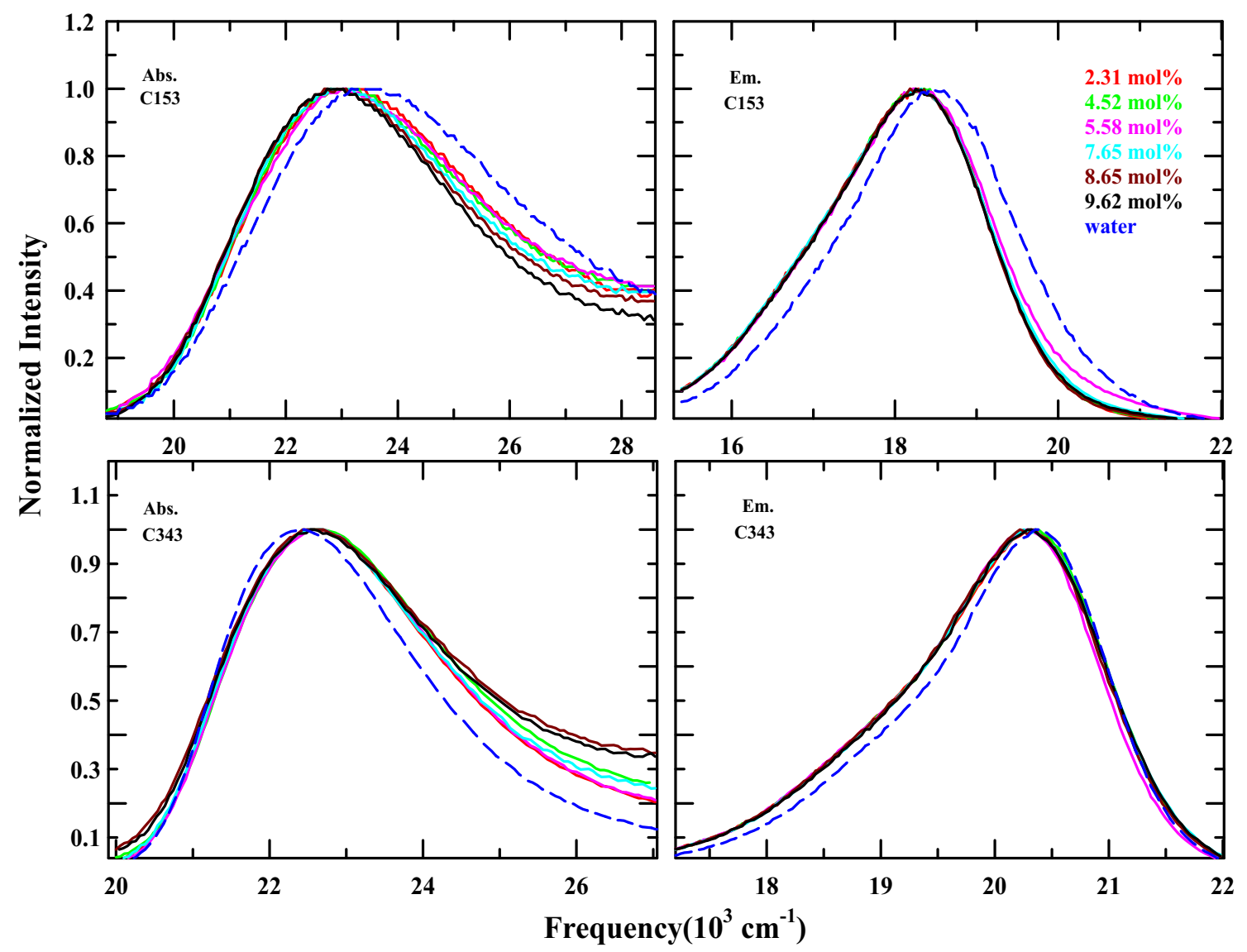

Figure 3. Absorption (left panels) and emission (right panels) spectra of $C 153$ and $C 343$ in water-xylitol mixtures at different concentration $(2.31,4.52,5.58,7.65,8.65,9.62$ mole \%) of xylitol at $298 \mathrm{~K}$. Blue broken line represents the absorption and emission spectra of $C 153$ and $C 343$ in neat water.

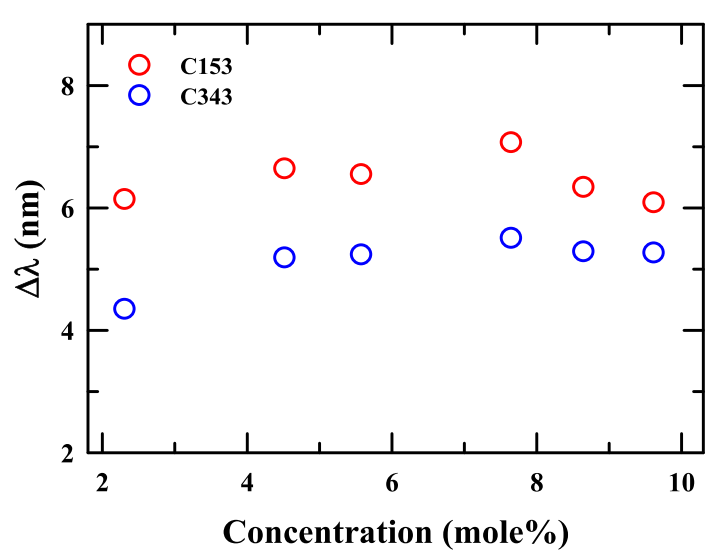

Figure 4. Excitation wavelength $\left(\lambda_{\text {exc }}\right)$ dependence of fluorescence emission peak wavelength difference (between red and blue end, $\Delta \lambda=\lambda_{\text {red,exc }}^{\text {em, eak }}-\lambda_{\text {blue,exc }}^{\text {em, peak }}$ ) for C153 (red circles) and C343 (blue circles) in water-xylitol mixtures at all xylitol mol\% studied. The excitation wavelengths are from $380 \mathrm{~nm}$ to $460 \mathrm{~nm}$ for C153, and $396 \mathrm{~nm}$ to $466 \mathrm{~nm}$ for C343 with $10 \mathrm{~nm}$ interval, scanning wavelengths that can cover $\sim 60 \%$ of the total intensity on both sides of the peak of the respective absorption spectra. these spectra fit to the multi-Debye model, and the fit parameters are summarized in Table 1. With temperature, the peak of $\varepsilon^{\prime \prime}$ shifts toward higher frequency. This is due to the lowering of solution viscosity with the rise in solution temperature leading to faster relaxation. As the slower relaxation becomes faster upon a rise in temperature with concomitant loss of amplitude, distinct relaxations may merge together at higher temperatures to produce total relaxations with fewer steps. This is the reason for two-step relaxation at higher temperatures for the lowest xylitol concentration studied here. Note also that the fastest relaxation component $\left(\tau_{3}\right)$ remains nearly insensitive to temperature variation whereas the other two show relatively stronger temperature dependence. This may be due to the limited frequency coverage of the present measurements which are unable to detect temperature-induced shortening of the fastest DR timescale.

Figure 2 shows the viscosity dependence of the average DR relaxation times $\left(\left\langle\tau_{\mathrm{DR}}\right\rangle\right)$ for aqueous xylitol solutions at the lowest (upper panel) and the highest (lower panel) concentrations. SED predictions with 


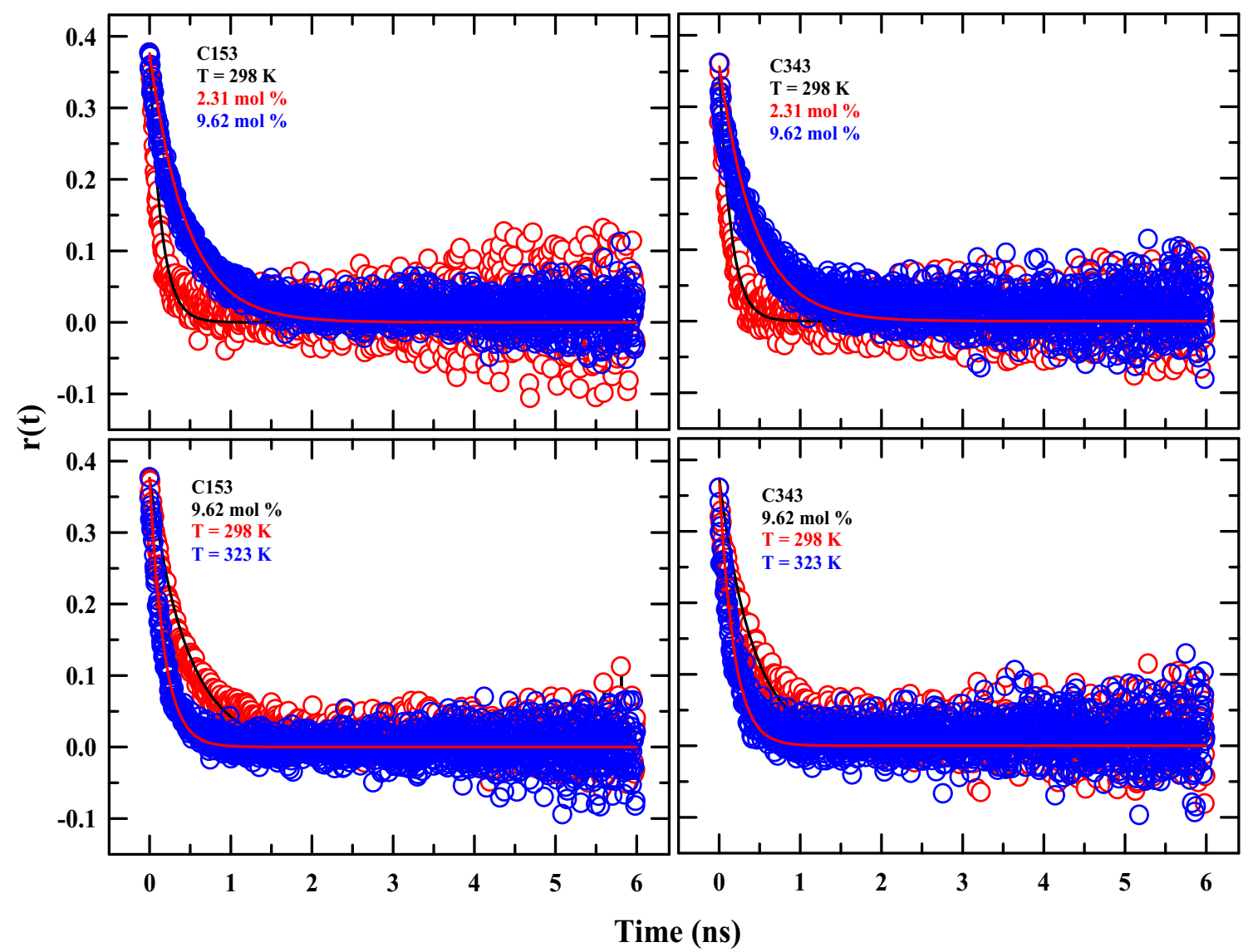

Figure 5. Representative time-resolved fluorescence anisotropy $(r(t))$ decays for $C 153$ (left panels) and $C 343$ (right panels) in water-xylitol mixture with 2.31 and $9.62 \mathrm{~mol} \%$ of xylitol (upper panel) and also at $298 \mathrm{~K}$ and $323 \mathrm{~K}$ (lower panel). Lines going through data denotes single exponential fits. (Residual of $r(t)$ decays are shown in Figure S2, Supplementary Information).

stick boundary condition for water and xylitol molecules using the experimental temperature dependent solution viscosity coefficients $(\eta$, see Table 2$)$ are also shown in these panels for comparison. Clearly, the SED predictions for xylitol are highly over-estimated relative to the average values from measurements at both the concentrations, whereas the calculations for water are strikingly close. Note these average times are the amplitude-weighted average of the DR relaxation times. A fit of these data to the expression, $\left\langle\tau_{\mathrm{DR}}\right\rangle=\mathrm{A} \eta^{\mathrm{p}}$, provides a value for the power $(\mathrm{p}=1.06)$ very similar to that for SED prediction $(\mathrm{p}=1)$ at $2.31 \mathrm{~mol} \%$. However, at $9.62 \mathrm{~mol} \%, \mathrm{p}=0.82$, which is smaller than unity. We, therefore, infer that these xylitol solutions are not strongly heterogeneous in the temperature range studied. In order to confirm this observation we have carried out both steady-state and timeresolved fluorescence measurements using hydrophobic (C153) and hydrophilic (C343) probes of comparable molecular volumes, ${ }^{53,68}$ results of which are presented later in this paper.
Figure 3 presents the UV-VIS absorption and steadystate fluorescence emission spectra for C153 and C343 in these aqueous xylitol solutions at $298 \mathrm{~K}$. For comparison, spectra of these solutes in neat water are also provided in the respective panels. It is quite evident that these spectra exhibit weak xylitol concentration dependence. In addition, C153 spectra in these aqueous solutions are slightly red-shifted than those in neat water, while the reverse (though faint) is seen for C343. This is probably because of their inherent preferences for solvation environments. The solution heterogeneity aspect is subsequently explored by monitoring the excitation wavelength dependence of the emission peak wavelengths for these solutes in these solutions at $298 \mathrm{~K}$. Figure 4 shows these results by showing a xylitol concentration dependence of the total dispersion of the peak emission wavelength ( $\left.\lambda_{\text {exc }}^{\text {em,peak }}\right)$ at a given concentration upon changing the excitation wavelength $\left(\lambda_{\text {exc }}\right)$ from blue to red across the corresponding absorption spectrum: $\Delta \lambda(\mathrm{c})=\lambda_{\text {red,exc }}^{\text {em,eak }}(\mathrm{c})-\lambda_{\text {blue,exc }}^{\text {em,peak }}(\mathrm{c})$. Clearly, these solutions are mildly heterogeneous as the total 


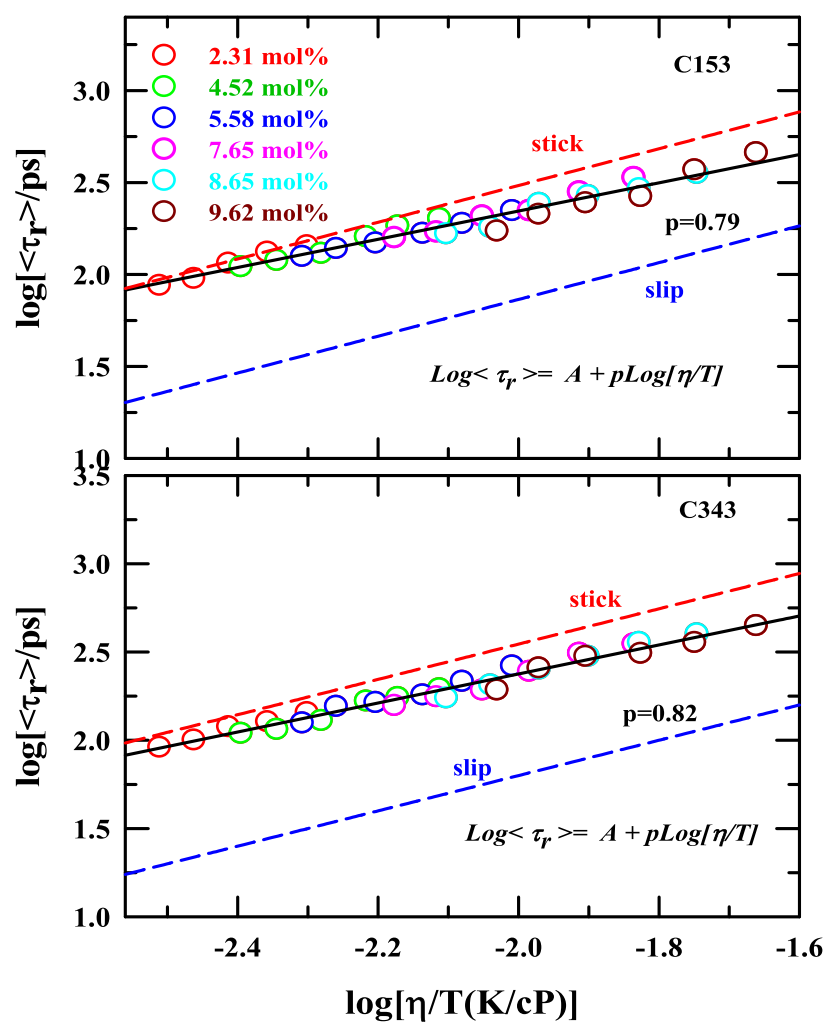

Figure 6. Viscosity coupling of rotation times $\left(\left\langle\tau_{r}\right\rangle\right)$ for C153 and C343 in water-xylitol mixtures at various xylitol concentrations are plotted. Temperature-dependent measured rotation times are shown as a function of temperature reduced viscosity $(\eta / T)$ in a log-log fashion. Lines through the data represent fits to the following expression: $\log \langle\tau\rangle=A+p \log [\eta / T]$. Broken lines represent the hydrodynamic (SED) predictions, $\tau_{r}^{S E D}=\left(V \eta / k_{B} T\right) f C$, where $V$-volume, $f$-shape factor and C-solutes-solvent coupling parameter. All these parameters are taken from Ref. 53 and Ref. 68 for C153 and C343, respectively.

dispersion of the peak emission wavelength $(\Delta \lambda)$ is not significant and remains limited only within $\sim 4-6$ nanometer for both the solutes.

Next, we follow the temperature and concentration dependence of dynamic fluorescence anisotropy, $r(t)$, for these solutes in these solutions. Figure 5 depicts the representative dependence where the collected data for the lowest and highest concentrations at room temperature (for concentration dependence), and for the highest concentration at $298 \mathrm{~K}$ and $323 \mathrm{~K}$ (for temperature dependence) are compared. Both these solutes show dependencies on these two solution parameters. As mentioned earlier, measured $r(t)$ decays for both the solutes fit adequately to single exponentials. This might be due to the limited temporal resolution employed $(\sim 85 \mathrm{ps})$ in the present measurements. ${ }^{53}$ Fits through these data are also shown in these panels, and the corresponding fit parameters summarized in Table 2. Average solute rotation times $\left(\left\langle\tau_{\mathrm{r}}\right\rangle\right)$ in this table is the relaxation time constant $\left(\tau_{\mathrm{r}}\right)$ associated with the underlying single-exponential decay function. This time constant, following the temperature dependence of viscosity, is becoming faster with temperature for all these solutions. The coupling to the viscosity of the solute rotation times in these solutions is then explored in Figure 6 where the measured $\left\langle\tau_{\mathrm{r}}\right\rangle$ are shown as a function of temperature-scaled viscosity, $\eta / T$, in a double-logarithmic fashion for both $\mathrm{C} 153$ (upper panel) and C343 (lower panel). Fit of these data to the viscosity dependence of the type, $\left\langle\tau_{r}\right\rangle \propto \eta^{p}$, then produces (represented by the solid line through the data) $\mathrm{p}$ values $(\sim 0.8)$ which are not too away from unity. Such values for the fraction power suggest the presence of mild temporal heterogeneity in these solutions. Note also that these $\mathrm{p}$ values are quite close to those obtained from DR measurements, and therefore inferences drawn from both these different experiments regarding solution dynamics corroborate well to each other. Combining steady-state fluorescence results with these relaxation measurements, one may then conclude that these solutions are not too spatially and temporally inhomogeneous.

If the relaxation times - be it from DR measurements or from dynamic fluorescence anisotropy experiments - follow closely the solution viscosity, then the activation energies extracted from the respective temperature dependent measurements should be agreeing well to each other. This exercise is undertaken next and the results are shown in Figure 7. Arrhenius-type temperature dependence is found for average rotational times for both the solutes in these solutions; so are for the average dielectric relaxation times and viscosity coefficients. Representative data for three different xylitol concentrations are shown for these observables along with the associated activation energies, $\mathrm{E}_{\mathrm{a}}$. Note the oneto-one correspondence between the activation energies at individual concentrations and the agreement among the concentration averaged activation energies, $\left\langle\mathrm{E}_{\mathrm{a}}\right\rangle_{\mathrm{c}}$, which ranges between $\sim 21 \mathrm{kJmol}^{-1}$ to $\sim 23 \mathrm{kJmol}^{-1}$. Such a good agreement among activation energies from different measurements originates from the overwhelming dominance of the frictional response of the system on these solution-phase relaxation processes in the temperature range studied, and the frictional resistance is nearly quantified by the macroscopic solution viscosity. This near-hydrodynamic coupling to solution viscosity (of relaxation dynamics) suggests mild spatiotemporal heterogeneity in these aqueous xylitol solutions at these temperatures. This is different from our earlier observation for other binary mixtures containing sugar. ${ }^{20}$ 

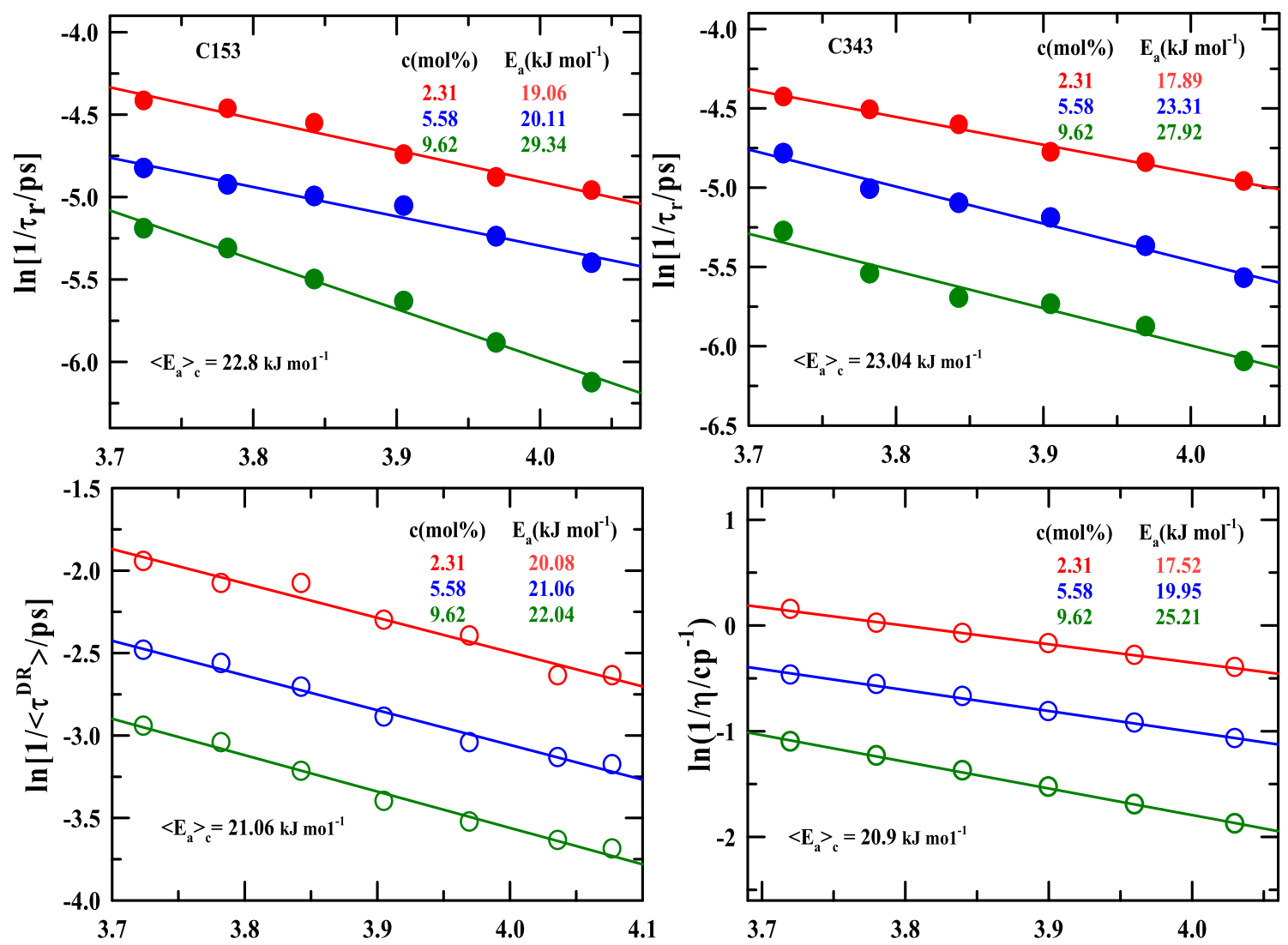

$10^{4} /\left[\mathrm{RT}\left(\mathrm{J} \mathrm{mol}^{-1}\right)\right]$

Figure 7. Arrhenius plot of $\ln \left(1 /\left\langle\tau_{r}\right\rangle\right)$ versus $1 /$ RT for $C 153$ (upper left panel) and $C 343$ (upper right panel) rotation times in water-xylitol mixtures (upper panel), and the same for the DR rotation times and viscosity coefficients (lower panel). Solid lines represent fit through the respective data sets.

\section{Conclusions}

In conclusion, the temperature dependent DR and fluorescence measurements suggest near-homogeneous solution structure and dynamics for these aqueous xylitol solutions in the temperature range studied. Multi-probe measurements do not indicate substantial concentration-dependent spectral shift, indicating no dramatic change in the overall polarity of the system in the presence of this poly-hydroxy alcohol. In addition, we do not find any evidence for cluster formation resulting from extensive H-bond interaction between water and xylitol molecules. In fact, the extent of viscosity coupling of probe rotation times observed in these solutions only indicates a mild heterogeneity. Measured DR timescales do not match the hydrodynamic predictions for molecular rotation of these species, leaving space for explanation in terms of $\mathrm{H}$-bond fluctuation dynamics and collective single particle reorientation relaxation. Extensive computer simulations are necessary for a microscopic understanding of the DR relaxation processes of these solutions, although the challenge here is to construct, at least qualitatively correctly, the inter- and intra-molecular interaction pair potentials. Such an effort is in progress.

\section{Supplementary Information (SI)}

Figures S1-S2 and Table S1 are available at www.ias.ac.in/ chemsci.

\section{References}

1. Vasilescu R, Ionescu A M, Mihai A, Carniciu S and Tîrgovişte C I 2011 Sweeteners and metabolic diseases: Xylitol as a new player Proc. Rom. Acad. Series B 2 125

2. Steinberg L M, Odusola F and Mandel ID 1992 Remineralizing potential, antiplaque and antigingivitis effects of xylitol and sorbitol sweetened chewing gum Clin. Prev. Dent. 1431

3. Miake Y, Saeki Y, Takahashi M and Yanagisawa T 2003 Remineralization effects of xylitol on demineralized enamel J. Electron. Microsc. 52471 
4. Makinen K K 2011 Sugar alcohol sweeteners as alternatives to sugar with special consideration of Xylitol Med. Princ. Pract. 20303

5. Foster-Powell K, Holt S H A, and Brand-Miller J C 2002 International table of glycemic index and glycemic load values: 2002 Am. J. Clin. Nutr. 765

6. Förster H, Boecker S and Walther A 1977 Use of xylitol as sugar substitute in diabetic children Fortschr Med. 95 99

7. Pepper T and Olinger P M 1988 Xylitol in sugar-free confections Food Technol. 4298

8. Birkhead D 1994 Cariologic aspects of xylitol and its use in chewing gum: A review Acta Odontol. Scand. 52116

9. Indra $\mathrm{S}$ and Biswas R 2016 Is dynamic heterogeneity of water in presence of a protein denaturing agent different from that in presence of a protein stabilizer? A molecular dynamics simulation study J. Chem. Sci. 1281943

10. Pradhan T, Ghoshal P and Biswas R 2008 Structural transition in alcohol-water binary mixtures: A spectroscopic study J. Chem. Sci. 120275

11. Indra S, Guchhait B and Biswas R 2016 Structural anomaly and dynamic heterogeneity in cycloether/water binary mixtures: Signatures from composition dependent dynamic fluorescence measurements and computer simulations J. Chem. Phys. 144124506

12. Gazi H A R and Biswas R 2011 Heterogeneity in binary mixtures of (water + tertiary butanol): Temperature dependence across mixture composition J. Phys. Chem. A 1152447

13. Sato T and Buchner R 2004 Dielectric relaxation processes in ethanol/water mixtures J. Phys. Chem. A 108 5007

14. Gheibi N, Saboury A A, Haghbeen K and MoosaviMovahedi A A 2006 The effect of some osmolytes on the activity and stability of mushroom tyrosinase $J$. Biosci. 31355

15. Kaushik J K and Bhat R 1998 Thermal stability of proteins in aqueous polyol solutions: Role of the surface tension of water in the stabilizing effect of polyols $J$. Phys. Chem. B $\mathbf{1 0 2} 7058$

16. Jain N K and Roy I 2009 Effect of trehalose on protein structure Protein Sci. 1824

17. Gekko K and Morikawa T 1981 Preferential hydration of bovine serum albumin in polyhydric alcohol-water mixtures J. Biochem. 9039

18. Mozhaev V V and Martinek K 1984 Structure-stability relationships in proteins: New approaches to stabilizing enzymes Enzyme Microb. Technol. 650

19. Dipaola G and Belleau B 1978 Polyol-protein interactions. Thermodynamical evidence for a selective solvation of glycerol and hexitols by aqueous P-lactoglobulin Can. J. Chem. 56848

20. Indra S and Biswas R 2016 How heterogeneous are trehalose/glycerol cryoprotectant mixtures? A combined time-resolved fluorescence and computer simulation investigation J. Phys. Chem. B 12011214

21. Nakanishi M and Nozaki R 2010 Dynamics and structure of hydrogen-bonding glass formers: Comparison between hexanetriol and sugar alcohols based on dielectric relaxation Phys. Rev. E 81041501

22. Psurek T, Maslanka S, Paluch M, Nozaki R and Ngai K L 2004 Effects of water on the primary and secondary relaxation of xylitol and sorbitol: Implication on the origin of the Johari-Goldstein relaxation Phys. Rev. E 70011503

23. Sidebottom D L 2007 Ultraslow relaxation of hydrogen-bonded dynamic clusters in glass-forming aqueous glucose solutions: A light scattering study Phys. Rev. E 76011505

24. Elamin K, Sjostorm J, Jansson H and Swenson J 2012 Calorimetric and relaxation properties of xylitol-water mixtures J. Chem. Phys. 136104508

25. Jansson H, Bergman R and Swenson J 2010 Hidden slow dynamics in water Phys. Rev. Lett. 104017802

26. Casalini R and Roland C M 2011 On the low frequency loss peak in the dielectric spectrum of glycerol J. Chem. Phys. 135094502

27. Yomogida Y, Minoguchi A and Nozaki R 2006 Ultraslow dielectric relaxation process in supercooled polyhydric alcohols Phys. Rev. E 73041510

28. Kaminski K, Wlodarczyk P, Hawelek L, Adrjanowicz K, Wojnarowska Z, Paluch M and Kaminska E 2011 Comparative dielectric studies on two hydrogen-bonded and van der Waals liquids Phys. Rev. E 83061506

29. Sidebottom D L and Tran T D 2010 Universal patterns of equilibrium cluster growth in aqueous sugars observed by dynamic light scattering Phys. Rev. E 82051904

30. Patkowski A, Gläser H, Kanaya T and Fischer E W 2001 Apparent nonergodic behavior of supercooled liquids above the glass transition temperature Phys. Rev. E 64 031503

31. Bergman R, Jansson H and Swenson J 2010 Slow Debye-type peak observed in the dielectric response of polyalcohols J. Chem. Phys. 132044504

32. Elamin K, Cazzato S, Sjostorm J, King S M and Swenson J 2013 Long-range diffusion in xylitol-water mixtures $J$. Phys. Chem. B 1177363

33. Rander D N, Joshi Y S, Kanse K S and Kumbharkhane A C 2016 Dielectric relaxation and hydrogen bonding interaction in xylitol-water mixtures using time domain reflectometry Indian Indian. J. Phys. 9067

34. Turton D A, Hunger J, Stoppa A, Hefter G, Thoman A, Walther M, Buchner R and Wynne K 2009 Dynamics of imidazolium ionic liquids from a combined dielectric relaxation and optical Kerr effect study: Evidence for mesoscopic aggregation J. Am. Chem. Soc. 13111140

35. Hunger J, Stoppa A, Schrödle S, Hefter G and Buchner R 2009 Temperature dependence of the dielectric properties and dynamics of ionic liquids ChemPhysChem $\mathbf{1 0}$ 723

36. Kaatze U and Giese K 1980 Dielectric relaxation spectroscopy of liquids: Frequency domain and time domain experimental methods J. Phys. E: Sci. Instrum. 13133

37. Barthel J, Bachhuber K, Buchner R and Hetzenauer H 1990 Dielectric spectra of some common solvents in the microwave region. Water and lower alcohols Chem. Phys. Lett. 165369

38. Mashimo S and Kuwabara S 1989 The dielectric relaxation of mixtures of water and primary alcohol J. Chem. Phys. 903292

39. Mukherjee K, Tarif E, Barman A and Biswas R 2017 Dynamics of a PEG based non-ionic deep eutectic solvent: Temperature dependence Fluid Phase Equilib. 448 22 
40. Mukherjee K, Das A, Choudhury S, Barman A and Biswas R 2015 Dielectric relaxations of (acetamide + electrolyte) deep eutectic solvents in the frequency window, $0.2 \leq v / \mathrm{GHz} \leq 50$ : Anion and cation dependence J. Phys. Chem. B 1198063

41. Indra $\mathrm{S}$ and Biswas R 2016 Are N-methyl groups of Tetramethylurea (TMU) Hydrophobic? A composition and temperature-dependent fluorescence spectroscopic investigation of TMU/water binary mixtures $\mathrm{J}$. Chem. Sci. 128753

42. Indra S and Biswas R 2015 Heterogeneity in (2butoxyethanol + water) mixtures: Hydrophobicityinduced aggregation or criticality-driven concentration fluctuations? J. Chem. Phys. 142204501

43. Das A and Biswas R 2015 Dynamic solvent control of a reaction in ionic deep eutectic solvents: Time-resolved fluorescence measurements of reactive and nonreactive dynamics in (choline chloride + urea) melts $J$. Phys. Chem. B 11910102

44. Guchhait B and Biswas R 2013 Ionic arrest of segmental motion and emergence of spatio-temporal heterogeneity: A fluorescence investigation of (polyethylene glycol + electrolyte) composites J. Chem. Phys. 138114909

45. Gazi H A R, Kashyap H K and Biswas R 2015 Solvent sorting in (mixed solvent + electrolyte) systems: Timeresolved fluorescence measurements and theory J. Chem. Sci. 12761

46. Guchhait B, Das S, Daschakraborty S and Biswas R 2014 Interaction and dynamics of (alkylamide + electrolyte) deep eutectics: Dependence on alkyl chain-length, temperature, and anion identity J. Chem. Phys. 140104514

47. Pradhan T and Biswas R 2007 Electrolyte-concentration and ion-size dependence of excited-state intramolecular charge-transfer reaction in (alkylamino)benzonitriles: Steady-state spectroscopic studies J. Phys. Chem. A 111 11514

48. Gazi H A R, Guchhait B, Daschakraborty S and Biswas R 2011 Fluorescence dynamics in supercooled (acetamide + calcium nitrate) molten mixtures Chem. Phys. Lett. $\mathbf{5 0 1} 358$

49. Guchhait B, Gazi H A R, Kashyap H and Biswas R 2010 Fluorescence spectroscopic studies of (acetamide + sodium/potassium thiocyanates) molten mixtures: Composition and temperature dependence J. Phys. Chem. B 1145066

50. Pradhan T, Gazi H A R, Guchhait B and Biswas R 2012 Excited state intramolecular charge transfer reaction in non-aqueous reverse micelles: Effects of solvent confinement and electrolyte concentration J. Chem. Sci. 124 355

51. Biswas R, Das A R, Pradhan T, Touraud D, Kunz W and Mahiuddin S 2008 Spectroscopic studies of catanionic reverse microemulsion: Correlation with the superactivity of horseradish peroxidase enzyme in a restricted environment J. Phys. Chem. B 1126620

52. Das A, Das S and Biswas R 2013 Fast fluctuations in deep eutectic melts: Multi-probe fluorescence measurements and all-atom molecular dynamics simulation study Chem. Phys. Lett. $\mathbf{5 8 1} 47$
53. Horng M L, Gardecki J A and Maroncelli M 1997 Rotational dynamics of coumarin 153: Time-dependent friction, dielectric friction, and other nonhydrodynamic effects J. Phys. Chem. A $\mathbf{1 0 1} 1030$

54. Das A, Das S and Biswas R 2015 Density relaxation and particle motion characteristics in a non-ionic deep eutectic solvent (acetamide + urea): Time-resolved fluorescence measurements and all-atom molecular dynamics simulations J. Chem. Phys. 142034505

55. Cross A J and Fleming G R 1984 Analysis of timeresolved fluorescence anisotropy decays Biophys. J. 46 45

56. Koley S, Kaur H and Ghosh S 2014 Probe dependent anomalies in the solvation dynamics of coumarin dyes in dimethyl sulfoxide-glycerol binary solvent: Confirming the local environments are different for coumarin dyes Phys. Chem. Chem. Phys. 1622352

57. Bottcher C J F and Bordewijk P 1978 Theory of Electrical Polarization 2nd edn. (Amsterdam, The Netherlands: Elsevier) Vol. 2

58. Bevington P R 1969 Data Reduction and Error Analysis for the Physical Sciences (New York: McGraw-Hill)

59. Maryott A A and Smith E R 1951 Table of Dielectric Constants of Pure Liquids National Bureau of Standards Circular 514, United States Department of Commerce

60. Buchner R, Barthel J and Stauber J 1999 The dielectric relaxation of water between $0{ }^{\circ} \mathrm{C}$ and $35^{\circ} \mathrm{C}$ Chem. Phys. Lett. 30657

61. Mukherjee K, Das S, Tarif E, Barman A and Biswas R 2018 Dielectric relaxation in acetamide + urea deep eutectics and neat molten urea: Origin of time scales via temperature dependent measurements and computer simulations J. Chem. Phys. 149124501

62. Dote J L, Kivelson D and Schwartz R N 1981 A molecular quasi-hydrodynamic free-space model for molecular rotational relaxation in liquids J. Phys. Chem. 85 2169

63. Das A, Biswas R and Chakrabarti J 2011 Dipolar solute rotation in a supercritical polar fluid J. Phys. Chem. A 115973

64. Das A, Biswas R and Chakrabarti J 2013 Dipolar solute rotation in ionic liquids, electrolyte solutions and common polar solvents: Emergence of universality Chem. Phys. Lett. 55836

65. Bottcher C J F 1946 Computation of the radius and the polarizability of a number of ions Rec. Trav. Chim. 65 14

66. Lucas M 1976 Size effect in transfer of nonpolar solutes from gas or solvent to another solvent with a view on hydrophobic behavior J. Phys. Chem. 80 359

67. Liu F F, Ji L, Zhang L, Dong X Y and Sun Y 2010 Molecular basis for polyol-induced protein stability revealed by molecular dynamics simulations J. Chem. Phys. 132 225103

68. Dutt G B and Ghanty T K 2003 Rotational diffusion of coumarins in electrolyte solutions: The role of ion pairs J. Phys. Chem. B $\mathbf{1 0 7} 3257$ 